\title{
CELLULOSE PHỦ NICKEL ÚNG DỤNG CHO QUÁ TRÌNH OXI HÓA ĐIỆN HÓA ETHANOL
}

\author{
TRAN THAO QUYNH NGAN ${ }^{1 *}$, PHAM HAI DINH ${ }^{2}$, NGUYEN DANG NAM ${ }^{3,4}$ \\ ${ }^{1}$ Khoa công nghệ hóa học, Trường Đại học Công nghiệp thành phố Hồ Chí Minh \\ ${ }^{2}$ Viện công nghệ Việt Hàn, Trường Đại học Công nghệ thành phố Hồ Chí Minh (HUTECH) \\ ${ }^{3}$ Viện Nghiên cúu Khoa học Co bản và Úng dụng, Trường Đại học Duy Tân \\ ${ }^{4}$ Khoa Môi trường và Công nghệ Hóa, Truờng Đại học Duy Tân
}

tranthaoquynhngan@iuh.edu.vn

\begin{abstract}
Tóm tắt. Vật liệu cellulose và nickel (Ni/cellulose) được ứng dụng tham gia vào phản ứng xúc tác điện hóa đối với ethanol. Cellulose được tổng hợp từ phế thải của nông nghiệp như vỏ cam ở miền nam Việt Nam thông qua phương pháp thủy nhiệt trong môi trường kiềm. Nickel đã được kết tủa thành công trên bề mặt cellulose bằng cách khử hydrazine hydrate. Hình thái học, tham số mạng và trạng thái bề mặt của hỗn hợp $\mathrm{Ni} /$ cellulose được xác định bằng phương pháp nhiễu xạ tia $\mathrm{X}(\mathrm{XRD})$, phổ hồng ngoại biến đổi Fourier (FTIR), kính hiển vi điện tử quét (SEM). Hoạt tính điện hóa của xúc tác Ni/cellulose cũng được khảo sát bằng phương pháp quét thế vòng tuần hoàn (cyclic voltammetry $-\mathrm{CV}$ ), quét thế tuyến tính (linear sweep voltammetry - LSV) và chronoamperometry - CA. Kết quả đặc tính cho thấy các hạt Ni kích thước nano phân bố không đồng đều trên mạch cellulose, xúc tác Ni/cellulose cho thấy hoạt tính điện hóa cao và ổn định đối với quá trình điện hóa ethanol. Mật độ dòng điện cực đại của xúc tác $\mathrm{Ni} / \mathrm{cell}^{\mathrm{a}}$ lolose là gần $1.2 \mathrm{~mA} . \mathrm{cm}^{-}$ ${ }^{2}$ khi xuất hiện $0,1 \mathrm{M}$ ethanol trong môi trường kiềm. Kết quả cho thấy rằng vật liệu $\mathrm{Ni} /$ cellulose hứa hẹn sẽ đóng gớp tích cực như tiếp cận mới cho lĩnh vực xúc tác điện hóa và pin nhiên liệu ethanol.
\end{abstract}

Từ khóa. điện hóa, ethanol, nickel

\section{NICKEL COVERED ON CELLULOSE TUBES FOR ETHANOL ELECTRO- OXIDATION REACTION}

\begin{abstract}
Nickel deposited cellulose material (Ni/cellulose) was applied for highly electro-oxidation reaction toward ethanol. Cellulose was collected from waste farm products such orange skin and shaddock cover in the south side of Vietnam via hydrothermal method in alkaline media. Nickel particle has been successfully precipitated on cellulose surface by hydrazine hydrate reduction. The micromorphology, lattice parameter and surface states of $\mathrm{Ni} /$ cellulose composite are determined by scanning electron microscopy (SEM), X-ray diffraction (XRD), energy dispersive X-ray (EDX) and Fourier-transform infrared spectroscopy (FTIR). The electrooxidation activity of Ni/cellulose catalyst was also investigated by cyclic voltammograms (CV), linear sweep voltammetry (LSV) and chronoamperograms (CA). Characterization results showed that Ni nanoclusters were uniformly distributed on cellulose tube, and $\mathrm{Ni} /$ cellulose catalyst was electrocatalytic activity and stability for ethanol electrooxidation. The maximum current density of $\mathrm{Ni} /$ cellulose catalyst was almost $1.2 \mathrm{~mA} \mathrm{~cm}^{-2}$ in alkaline media with $0.1 \mathrm{M}$ ethanol. Ni/cellulose composite would be a promising approach toward ethanol electro-oxidation reaction and fuel cell.
\end{abstract}

Keyword. Electro-oxidation, ethanol, nickel, cellulose

\section{GIÓ́I THIỆU}

Sự gia tăng dân số, đô thị hóa và mức sống tăng cao đã làm gia tăng nhanh chóng mức tiêu thụ năng lượng toàn cầu và gánh nặng môi trường trong nửa thế kỷ qua. Tiêu thụ năng lượng được dự kiến sẽ tiếp tục tăng mạnh trong tương lai. Thêm vào đó, sự cạn kiệt nguồn nhiên liệu hóa thạch như than đá, dầu mỏ... tạo động lực thúc đẩy phát triển việc nghiên cứu và ứng dụng của các nguồn nguyên liệu thay thế [1]. Các nguồn nguyên liệu bền vững và có khả năng tái tạo như năng lượng mặt trời, gió hay nhiên liệu xanh như hydro, methanol... đang được quan tâm thay thế cho nguồn nhiên liệu hóa thạch [2]. Ethanol, một hợp chất hữu cơ phổ biến, là nguyên liệu cũng như sản phẩm của nhiều ngành công nghiệp như thực phẩm, mỹ 
phẩm...[3]. Do tính phổ biến, không độc hại và không cháy nổ, ethanol thể hiện là một nhiên liệu hữu cơ đang được quan tâm nghiên cứu và ứng dụng. Trong giới hạn đề tài này, oxi hóa điện hóa ethanol được trọng tâm nghiên cứu như một đóng góp cho ứng dụng xa hơn trong việc phát triển ethanol như nhiên liệu xanh tìm năng.

Trong những thập niên gần đây, các nghiên cứu nhằm nâng cao hiệu suất cho phản ứng oxi hóa điện hóa đối với ethanol đang được quan tâm [4]. Hàng loạt kim loại và hợp chất kim loại có vai trò quan trọng trong ứng dụng như là xúc tác trong lĩnh vực này [5]. Các kim loại hiếm như vàng $(\mathrm{Au})[6]$, bạc $(\mathrm{Ag})[7]$, và platinum $(\mathrm{Pt})[6,8]$ đã thể hiện khả năng xúc tác hiệu quả do khả năng giảm năng lượng hoạt hóa cũng như bền với môi trường của những phản ứng điện hóa. Tuy nhiên yếu tố kinh tế đã trở thành điểm hạn chế trong việc ứng dụng của các kim loại trong phân lớp này. Gần đây, nickel [9-11] cùng một số kim loại chuyển tiếp khác như Co [12], Fe [13] , Cu [14] ... thể hiện tiềm năng cao cho ứng dụng xúc tác và cũng như là xúc tác điện hóa. Mặc dù hiệu quả xúc tác cao, giá trị kinh tế ổn định, nhưng các kim loại thuộc nhóm này dễ bị ăn mòn hay ngộ độc do môi trường phản ứng. Vì vậy, hàng loạt các hợp chất từ nhóm xúc tác này đang được nghiên cứu để nâng cao hiệu quả và khắc phục các hạn chế tồn tại.

Bằng việc kết hợp với các vật liệu khác như cacbon, $C N T$, graphene ..., các vật liệu mới từ nickel thay đổi hình dạng, kích thước và các tính chất của nó [15]. Trong đó, cellulose là một trong những hợp chất từ cacbon có cấu trúc phù hợp hố trợ tổng hợp xúc tác nickel. Đặc biệt, cellulose được thu thập dễ dàng từ nhiều nguồn phế phẩm nhưng: vỏ, thân, lá cây... Trong tình hình Việt Nam hiện tại, vỏ cam được thải bỏ trong công nghiệp thực phẩm có thể được xem là nguồn nguyên liệu tái chế dồi dào cho thu thập cellulose. Cellulose được biết đến như là một polyme tự nhiên mạch thẳng được cấu tạo từ các đơn vị $\beta, 1-4$, Danhydro-glucopyranose, các đơn vị này liên kết với nhau bởi liên kết $(1,4)$-glycosidic . Nhờ vào cấu tạo dạng ống tự nhiên, các hạt nickel dễ dàng kết tủa lên cấu trúc cellulose hình thành cấu tạo 2 chiều (2D), vì thế hình dạng và đặc tính xúc tác nickel từ đó thay đổi. Gần đây, cellulose được chú ý nhiều như một chất hỗ trợ nhằm giảm kích thước hạt, tăng diện tích tiếp xúc cũng như tính xúc tác của kim loại [16].

Trong giới hạn nghiên cứu, cellulose dùng trong quá trình tổng hợp xúc tác Ni/cellulose được lấy từ vỏ cam sành, được thu thập từ nguồn phế thải thực phẩm. Cellulose được tách ra từ vỏ cam có kích thước $~ 500 \mathrm{~nm}$ bằng phương pháp nghiền, rây thành bột mịn, tinh dầu và các chất hữu cơ khác được loại bỏ bằng phương pháp thủy phân trong môi trường kiềm. $\mathrm{Ni} /$ cellulose được tổng hợp bằng phương pháp thủy nhiệt ở nhiệt độ $200{ }^{\circ} \mathrm{C}$ trong môi trường glycerol. Các tính chất hóa lý của cellulose và $\mathrm{Ni} /$ cellulose được kiểm tra bằng phương pháp đo nhiễu xạ tia X (XRD), phổ hồng ngoại biến đổi Fourier (FTIR), kính hiển vi điện tử quét (SEM). Các phương pháp quét thế tuần hoàn cyclic voltammetry $(\mathrm{CV})$, quét điện thế tuyến tính linear sweep voltammetry (LSV) và chronoamperometry $(\mathrm{CA})$ được sử dụng để kiểm tra khả năng xúc tác điện hóa của vật liệu được tổng hợp.

\section{THỰC NGHIỆM}

\section{a. Hóa chất}

Vỏ cam được thu gom từ nguồn rác thải thực phẩm trên địa bàn thành phố Hồ Chí Minh. Các hóa chất khác như natri hydroxit $(\mathrm{NaOH})$, axit clohydric $(\mathrm{HCl})$, ethanol $(\mathrm{EtOH})$, nickel clorua $\left(\mathrm{NiCl}_{2} \cdot 6 \mathrm{H}_{2} \mathrm{O}\right)$, hydrazin hydrate $\left(\mathrm{N}_{2} \mathrm{H}_{4}\right)$ và kali hydroxit $(\mathrm{KOH})$ được sử dụng theo chuẩn phân tích và mua từ các hãng của Trung Quốc.

\section{b. Tách cellulose}

Cellulose được tách từ vỏ cam bằng phương pháp thủy phân trong môi trường kiềm theo các công bố trước đó [16-18]. Quy trình tách cellulose và tổng hợp Ni/cellulose được miêu tả như hình 1 . Vỏ cam sau khi thu gom được vệ sinh, sấy khô và nghiền thành bột mịn có kích thước nhỏ hơn $0,125 \mu \mathrm{m}$ bằng máy nghiền và hệ thống rây Retsch - Germany. $20 \mathrm{~g}$ vỏ cam được khuấy đều trong $300 \mathrm{ml} \mathrm{NaOH} 20 \%$ (wt.) trong 1 giờ để được hỗn hợp đồng nhất. Hồn hợp đồng nhất này được gia nhiệt lên $120^{\circ} \mathrm{C}$ trong 2 giờ để thực hiện quá trình thủy phân tách cellulose khỏi những hợp chất khác có trong vỏ cam. Chất rắn thu được sau quá trình thủy phân trong môi trường kiềm được loại bỏ lignin còn sót lại bằng cách khuấy trong $360 \mathrm{ml}^{\mathrm{H}} \mathrm{H}_{2} \mathrm{O}_{2}(0.5 \%$ wt.) 2 giờ ở $80^{\circ} \mathrm{C}$. Sau quá trình loại lignin, chất rắn tiếp tục được rửa lần lượt bằng $\mathrm{HCl} 0.1 \mathrm{M}$ và ethanol $80 \%$ ở nhiệt độ $80^{\circ} \mathrm{C}$ để loại bỏ thành phần muối, kim loại và các hợp chất hữu cơ còn sót lại sau quá trình. Sau quá trình rửa, bột cellulose màu trắng xám thu được sau khi sây khô ở $60{ }^{\circ} \mathrm{C}$. 


\section{c. Tổng hơp Ni/cellulose}

Composite $\mathrm{Ni} /$ cellulose được tổng hợp bằng phương pháp thủy nhiệt. Hỗn hợp gồm $0,135 \mathrm{~g} \mathrm{NiCl}_{2} \cdot 6 \mathrm{H}_{2} \mathrm{O}$ và $50 \mathrm{ml}$ glixerol được khuấy đều trong 30 phút để đạt được dung dịch màu xanh. $0,5 \mathrm{~g}$ cellulose được thêm vào hô̂n hợp trên và tiếp tục khuấy trong 24 giờ để đạt được một hồn hợp đồng nhất. Sau đó, hỗn hợp đồng nhất này được cho thêm vào $5 \mathrm{ml}$ dung dịch hydrazine $80 \%$ khuấy đều 5 phút và chuyển vào bình phản ứng thủy nhiệt thực hiện phản ứng thủy nhiệt ở $200{ }^{\circ} \mathrm{C}$ trong vòng 8 giờ. Một hỗn hợp composite $\mathrm{Ni} /$ cellulose màu xám đen thu được từ sản phẩm của quá trình thủy nhiệt được lọc, rửa và sấy khô ở $60^{\circ} \mathrm{C}$.

\section{d. Tính chất vật liệu}

Cấu trúc của mẫu cellulose và composite $\mathrm{Ni}$ /cellulose đã tổng hợp được xác định bằng phương pháp nhiễu xạ tia X (XRD; Shimadzu - LabX XRD 6100), quang phổ chuyển đổi hồng ngoại (Fourier Fourier transform infrared spectroscopy - FTIR; Jasco FTIR-4700). Hình dạng và kích thước mẫu được khảo sát bằng phương pháp kính hiển vi điện tử quét (scanning electron microscopy-SEM; Hitachi S4800 - Japan). Tính chất điện hóa đối với ethanol của mẫu được khảo sát bằng phương pháp quét thế tuần hoàn cyclic voltammetry $(\mathrm{CV})$, quét điện thế tuyến tính linear sweep voltammetry (LSV) và chronoamperometry (CA) (CV) (VSP, biologic) với hệ 3 điện cực. Hệ 3 điện cực sử dụng trong nghiên cứu hoạt tính xúc tác điện hóa của điện cực gồm: điện cực làm việc gồm mẫu được phủ lên điện cực lõi Pt đường kính $3 \mathrm{~mm}$; điện cực lưới Pt và điện cực tham khảo $\mathrm{Ag} / \mathrm{AgCl}$. Dung dịch sử dụng cho quá trình khảo sát điện hóa là dung dịch kiềm $\mathrm{KOH} 0,1 \mathrm{M}$.

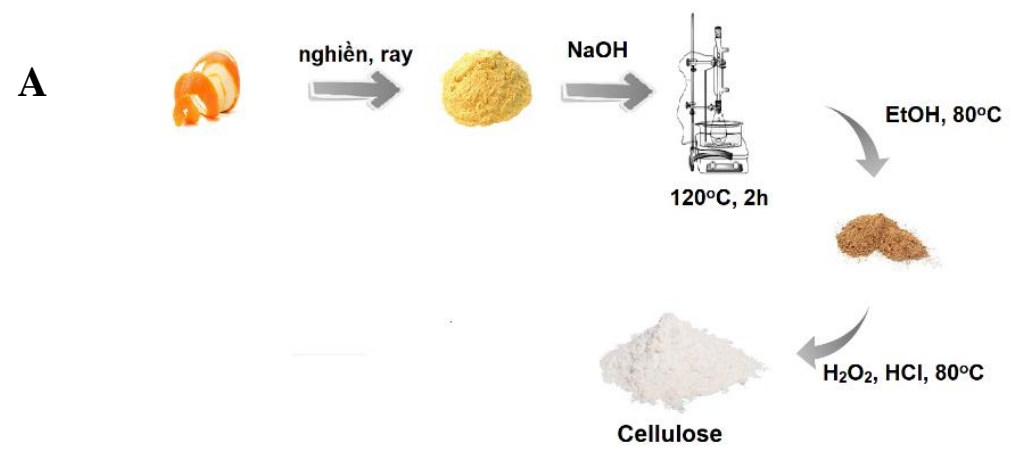

B
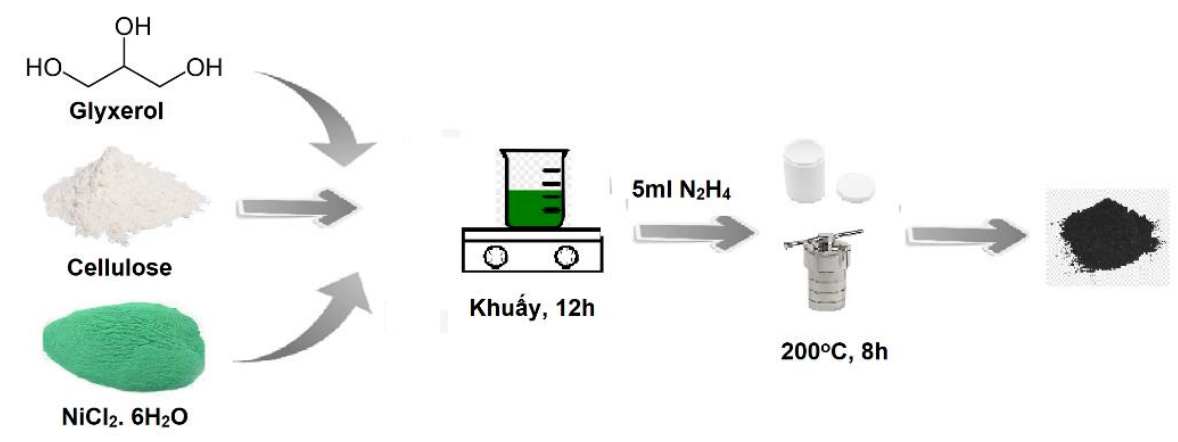

Hình 1. Quy trình tách cellulose ra khỏi vỏ cam (A) và tổng hợp composite Ni/cellulose (B)

\section{KẾT QUẢ VÀ THẢO LUẬN}

\section{a. Cấu trúc vật liệu}

Hình 2 là kết quả nhiễu xạ tia $X(X R D)$ và FTIR của các mẫu cellulose và Ni/cellulose đã được tổng hợp. Kết quả XRD của mẫu cellulose (hình $2 \mathrm{~A}$ ) với cấu trúc vô định hình có 2 đỉnh ở vị trí góc $2 \theta$ là $19.4^{\circ}$ và $21.6^{\circ}$ tương ứng với các mặt (110) và (002) của cellulose, phù hợp với phổ XRD của cellulose đã được công bố ở các kết quả trước đây $[16,19]$. Mẫu Ni/cellulose có kết quả XRD đồng thời của các đỉnh đặc trưng của cả cellulose và tinh thể nickel. Đối với kết quả XRD của composite Ni/cellulose, ngoài hai đỉnh (110) và (002) của cellulose như đã phân tích ở trên, cấu trúc lập phương của nickel $(\mathrm{Fm}-3 \mathrm{~m}(225))$ được xác định từ các đỉnh ở các vị trí góc $2 \theta$ là $44.2^{\circ}, 51.7^{\circ}, 76.3^{\circ}$ tương ưng với các mặt phẳng $(111)$, (200) và (220) phù hợp với cấu trúc đã được công bố trước đó [20,21] (JCPDS 04-0850). Ngoài ra, các đỉnh ở vị trí 
góc $2 \theta$ là $29.6^{\circ}, 36.3^{\circ}, 39.9^{\circ}, 48.6^{\circ}$ cho thấy sự hiện diện của nickel carbide (NiCx) (JCPDS 45-0979)[22], chứng tỏ việc phủ nickel lên bề mặt cellulose.

Kết quả FTIR của cellulose và composite Ni/cellulose được thể hiện trong hình 2B. Các đỉnh ở $2902 \mathrm{~cm}^{-1}$ và $975-1155 \mathrm{~cm}^{-1}$ lần lượt đặc trưng cho dao động của liên kết $\mathrm{C}-\mathrm{H}$ và $\mathrm{C}-\mathrm{O}$ trong mạch cellulose. Dãi rộng ở $3368.4 \mathrm{~cm}^{-1}$ thể hiện liên kết của nhóm $\mathrm{O}-\mathrm{H}$ và $1403.8 \mathrm{~cm}^{-1}$ tương ứng với liên kết carbonyl O-H. Liên kết $\mathrm{C}=\mathrm{C}$ trong cấu trúc cellulose cũng được thể hiện rõ qua dao động ở vị trí đỉnh $1647.1 \mathrm{~cm}^{-1}$. Các liên kết trong cấu trúc cellulose đã được thể hiện rõ qua các đỉnh đặc trưng trong kết quả FTIR như kết quả trước đó đã công bố [19]. Vì vậy, kết quả XRD và FTIR chỉ ra việc tách thành công cellulose ra khỏi vỏ cam bằng phương pháp thủy phân trong môi trường kiềm trong điều kiện nghiên cứu. Khi xuất hiện nickel kết tủa trên bề mặt cellulose, các đỉnh đặc trưng của cellulose vẫn xuất hiện trong kết quả FTIR của composite $\mathrm{Ni} /$ cellulose, tuy nhiên, cường độ đã giảm mạnh. Như vậy nickel bao phủ trên bề mặt cellulose đã làm giảm tính hiệu của các dao động trong mạch cellulose.
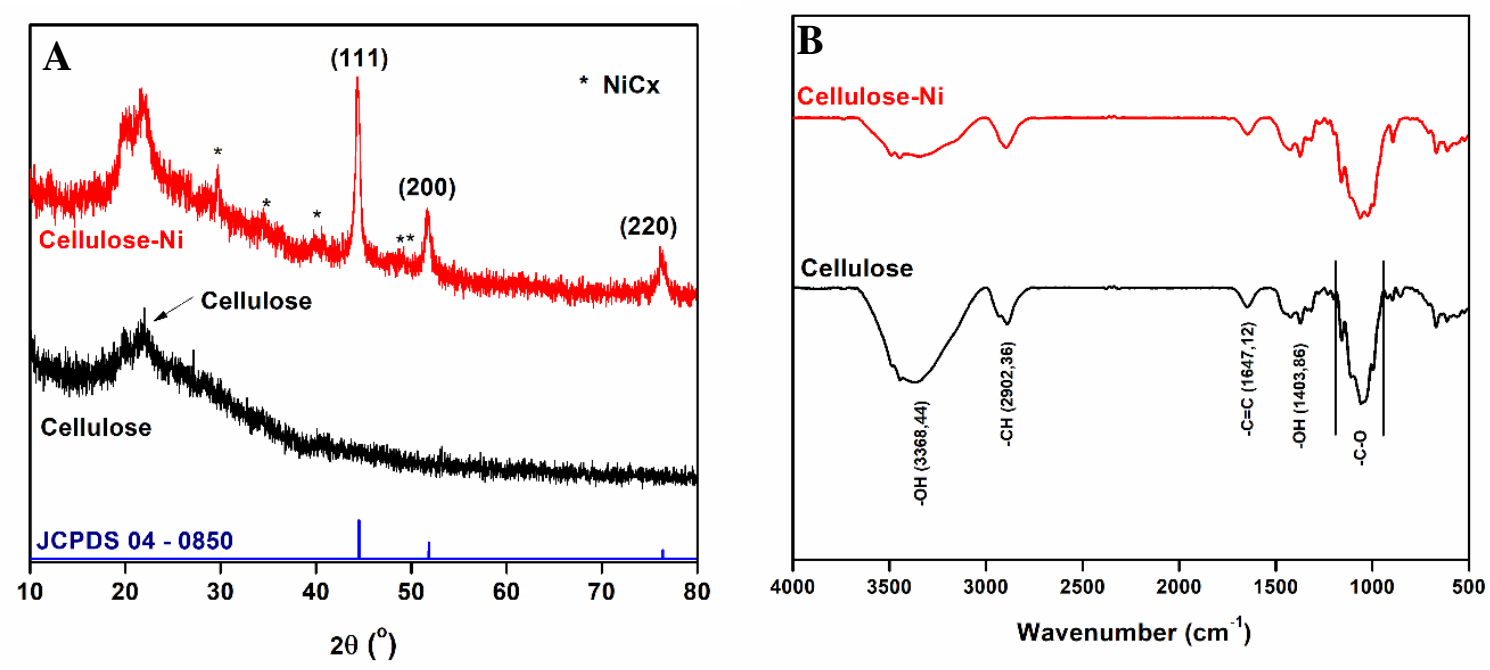

Hình 2. Kết quả XRD $(\mathrm{A})$ và FTIR $(\mathrm{B})$ của mẫu cellulose và Ni/cellulose.

Hình dạng và kích thước của cellulose và composite Ni/cellulose được thể hiện rõ qua kết quả SEM (hình 3). Kết quả SEM cho thấy cellulose được tách từ vỏ cam có dạng bó, gồm nhiều sợi của các chuỗi $\left(\mathrm{C}_{6} \mathrm{H}_{10} \mathrm{O}_{5}\right)$ n có đường kính $~ 100 \mathrm{~nm}$. Do sơ chế trước khi tiến hành tách loại cellulose, chiều dài các chuỗi cellulose được khống chế từ 500 - $1000 \mathrm{~nm}$ (hình 3A). Bề mặt không đồng nhất của chuỗi cellulose hỗ trợ cho nickel phủ lên trên. Hình $3 \mathrm{~B}$ cho thấy composite Ni/cellulose là một hỗn hợp gồm có nickel bao phủ trên bề mặt cellulose. Các ống cellulose được các hạt nickel có kích thước $\sim 50 \mathrm{~nm}$ kết tủa trên bề mặt. Sự xuất hiện của cellulose sẽ giảm khả năng kết tụ của các hạt nickel khi hình thành.

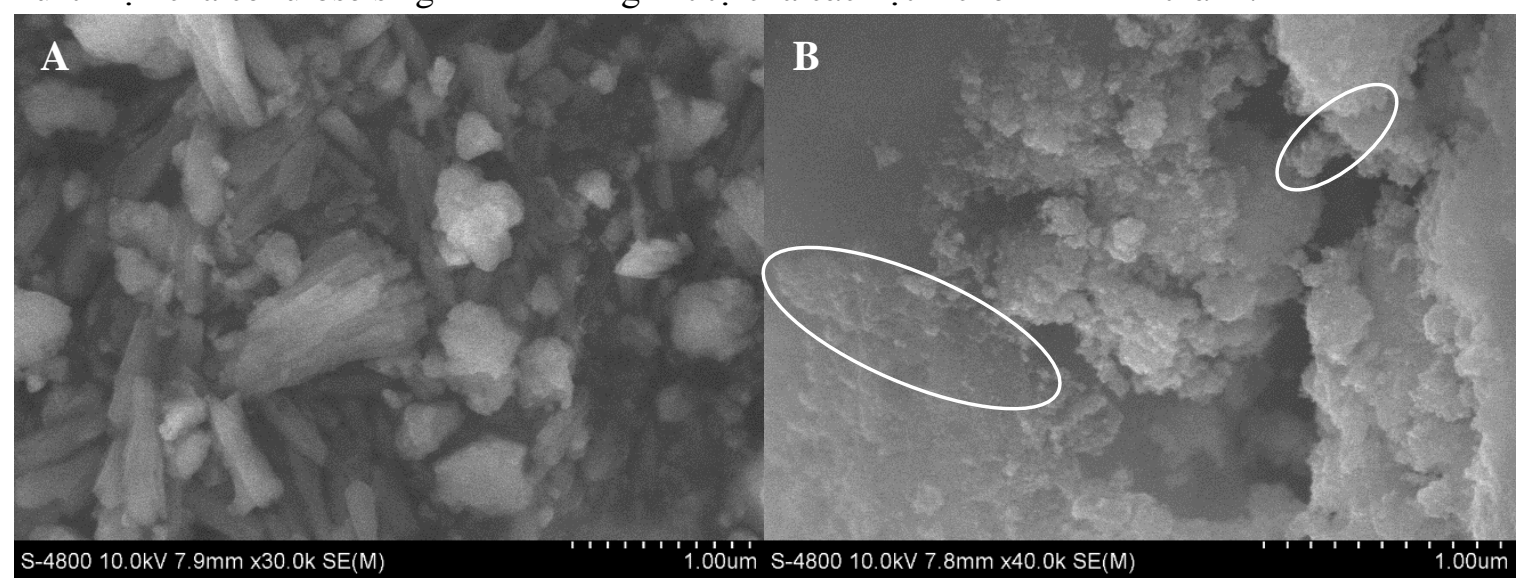

Hình 3. Hình SEM của cellulose $(\mathrm{A})$ và $\mathrm{Ni} /$ cellulose $(\mathrm{B})$

b. Tính chất oxi- hóa điện hóa ethanol

Hình 4 là kết quả điện thế quét (linear sweep voltage - LSV) của mẫu cellulose trong môi trường KOH $0.1 \mathrm{M}$ và $\mathrm{Ni} /$ cellulose trong $\mathrm{KOH} 0.1 \mathrm{M}$ và $\mathrm{KOH} 0.1 \mathrm{M}$ + ethanol $0.1 \mathrm{M}$. Các mẫu được quét trên dải thế từ 
0 - $0.8 \mathrm{~V}$ với vận tốc quét $20 \mathrm{mV} / \mathrm{s}$ trong môi trường kiềm $(\mathrm{KOH} 0.1 \mathrm{M})$ với các điều kiện có sự hiện diện hay không có sự hiện diện của ethanol. Cellulose cho kết quả hầu như không tham gia quá trình điện hóa (đường màu xanh), tín hiệu dòng điện đo được $\sim 0 \mathrm{~mA}$ trong môi trường phân tích. Trong cùng một điều kiện phân tích, vật liệu composite Ni/cellulose thể hiện tín hiệu điện tại hiệu điện thế $~ 0.7 \mathrm{~V}$ là do chuyển trạng thái của cặp oxi hóa $\mathrm{Ni}^{2+} / \mathrm{Ni}^{3+}$ trên bề mặt vật liệu khi nickel tiếp xúc $\mathrm{OH}^{-}$trong môi trường kiềm (phương trình 1). Quá trình chuyển trạng thái oxi hóa của $\mathrm{Ni}^{2+} / \mathrm{Ni}^{3+}$ cung cấp electron trên điện cực làm tăng cường độ dòng điện [11]. Khi có sự hiện diện của ethanol, mật độ dòng điện tăng đáng kể (đường màu đỏ). Oxi hóa điện hóa của ethanol trên bề mặt xúc tác $\mathrm{Ni}^{2+} / \mathrm{Ni}^{3+}$ cung cấp lượng lớn electron làm tăng mật độ dòng điện (phương trình 2) $[13,15,16]$. Quá trình oxi hóa ethanol được ghi nhận bắt đầu từ hiệu điện thế $\sim 0.35 \mathrm{~V}$. Theo đó, quá trình chuyển trạng thái trên bề mặt xúc tác được dẫn ra như sau:

$\mathrm{Ni}^{2+} \Leftrightarrow \mathrm{Ni}^{3+}+\mathrm{e}$

Ethanol $=>$ sản phẩm $+\mathrm{e}$

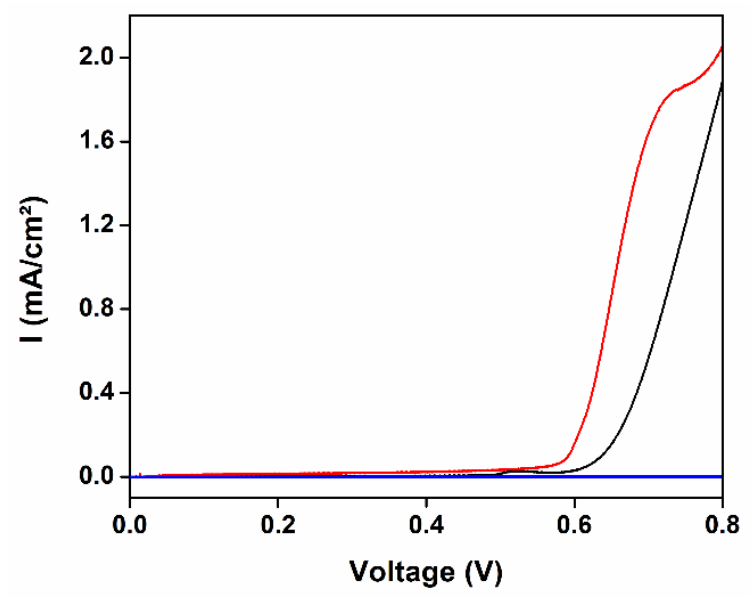

Hình 4. Kết quả LSV của mẫu cellulose được phân tích trong KOH $0.1 \mathrm{M}$ (màu xanh) và mẫu Ni/cellulose lần lượt phân tích trong $\mathrm{KOH} 0.1 \mathrm{M}$ (đường màu đen) và $\mathrm{KOH} 0.1 \mathrm{M}+$ ethanol $0.1 \mathrm{M}$ (đường màu đỏ) với thế quét từ $0-0.8 \mathrm{~V}$ (vs. $\mathrm{Ag} / \mathrm{AgCl}(\mathrm{NaCl}$ sat.)), tốc độ quét $20 \mathrm{mV} / \mathrm{s}$.

Hình 5 là kết quả quét điện thế chu kỳ (cyclic voltammetry - $\mathrm{CV}$ ) của mẫu Ni/cellulose lần lượt trong $\mathrm{KOH}$ $0.1 \mathrm{M}$ (đường màu đen) và $\mathrm{KOH} 0.1 \mathrm{M}+$ ethanol $0.1 \mathrm{M}$ (đường màu đỏ). Mẫu $\mathrm{Ni} / \mathrm{cellul}$ lose được quét theo chu kỳ với hiệu điện thế được giới hạn từ $0-0.8 \mathrm{~V}$ và tốc độ quét $20 \mathrm{mV}$. Trong trường hợp chỉ có $\mathrm{KOH}$, sự chuyển điện hóa tuần hoàn của $\mathrm{Ni}^{2+} / \mathrm{Ni}^{3+}$ hình thành 2 đỉnh của cường độ dòng ở hiệu điện thế $0.5 \mathrm{~V} /$ $0.42 \mathrm{~V}$ như đã thảo luận (phương trình 1 ). Khi xuất hiện ethanol, phản ứng hóa học oxi hóa ethanol cung cấp electron làm tăng cường độ dòng điện (phương trình 2) đối với quá trình chuyển $\mathrm{Ni}^{2+}=>\mathrm{Ni}^{3+}$. Khi chu kỳ ngược diễn ra, tức là quá trình $\mathrm{Ni}^{3+}=>\mathrm{Ni}^{2+}, \mathrm{Ni}^{3+}$ được cung cấp electron từ điện cực nguồn và electron sinh ra từ phản ứng oxi hóa ethanol, do đó cường độ dòng điện vẫn duy trì (so sánh với trường hợp không có ethanol). Tương tự, kết quả đo cường độ dòng điện (chronoamperometry -CA) trên các vật liệu tại hiệu điện thế $0.7 \mathrm{~V}$ trong 10 phút phù hợp với kết quả LSV và $\mathrm{CV}$ đã thảo luận. Tại hiệu điện thể $0.7 \mathrm{~V}$, cường độ dòng điện của hệ chứa vật liệu cellulose cho thấy rất thấp tín hiệu dòng điện (đường màu xanh) trong $\mathrm{KOH} 0.1 \mathrm{M}$ thể hiện không có phản ứng điện hóa xảy ra trên bề mặt cellulose. Vật liệu Ni/cellulose duy trì cường độ dòng điện lần lượt là $\sim 0.5 \mathrm{~mA} / \mathrm{cm}^{2}$ và $\sim 1.2 \mathrm{~mA} / \mathrm{cm}^{2}$ tại hiệu điện thế $0.7 \mathrm{~V}$ trong thời gian hoạt động ổn định 10 phút với dung môi là $\mathrm{KOH} 0.1 \mathrm{M}$ và $\mathrm{KOH} 0.1 \mathrm{M}$ chứa ethanol $0.1 \mathrm{M}$ (hình 6). Từ những kểt quả cho thấy tính khả thi của việc sử dụng vật liệu composite $\mathrm{Ni}$ /cellulose cho phản ứng xúc tác điện hóa ethanol trong môi trường kiềm. Cấu tạo dạng ống của cellulose hứa hẹn sẽ là vật liệu hỗ trợ tốt để tổng hợp xúc tác có cẩu trúc 2 chiều. Tuy nhiên vấn đề, độ bền của cellulose khi làm việc trong môi trường kiềm và khả năng dẫn electron thấp của cellulose được nhận thấy là những hạn chế khi làm việc với vật liệu composite $\mathrm{Ni} /$ cellulose. 


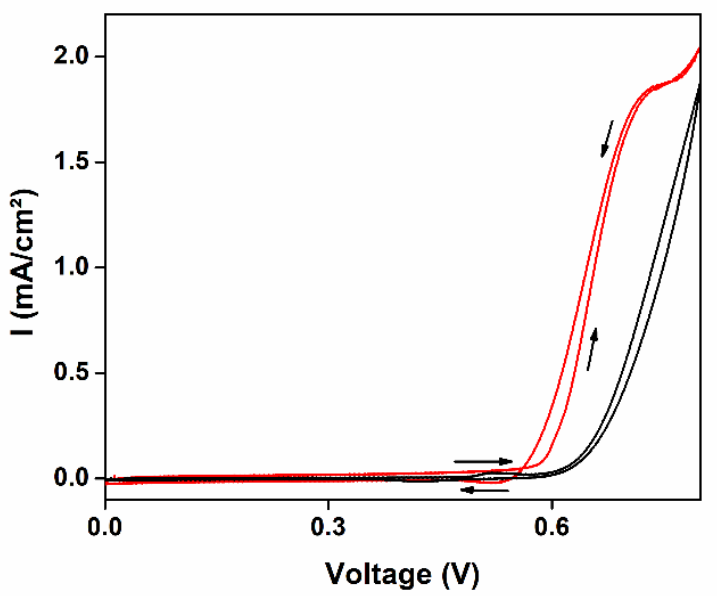

Hình 5. Kết quả $\mathrm{CV}$ của mẫu Ni/cellulose lần lượt phân tích trong $\mathrm{KOH} 0.1 \mathrm{M}$ (đường màu đen) và $\mathrm{KOH}$ $0.1 \mathrm{M}+$ ethanol $0.1 \mathrm{M}$ (đường màu đỏ) với thế quét từ $0-0.8 \mathrm{~V}$ (vs. $\mathrm{Ag} / \mathrm{AgCl}(\mathrm{NaCl}$ sat.)), tốc độ quét 20 $\mathrm{mV} / \mathrm{s}$.

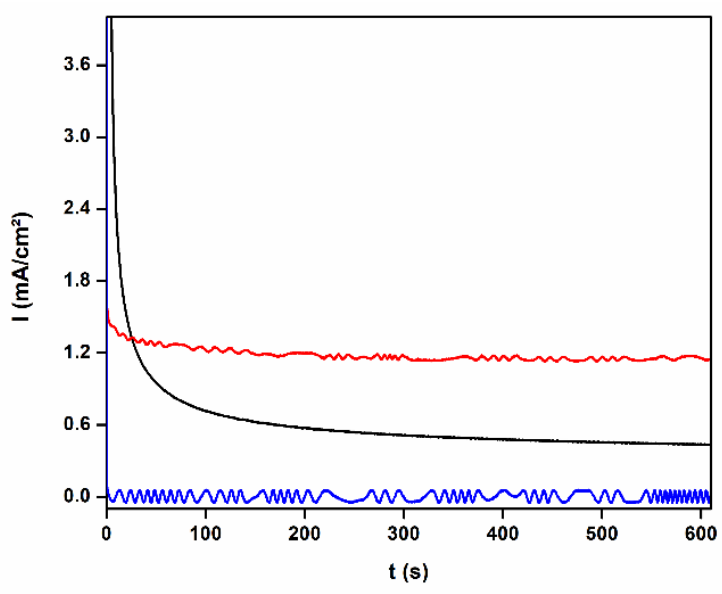

Hình 6. Kết quả CA của mẫu cellulose được phân tích trong môi trường $\mathrm{KOH} 0.1 \mathrm{M}$ (đường màu xanh) và $\mathrm{Ni} /$ cellulose lần lượt được phân tích trong môi trường $\mathrm{KOH} 0.1 \mathrm{M}$ (đường màu đen) và $\mathrm{KOH} 0.1 \mathrm{M}$ chứa ethanol $0.1 \mathrm{M}$ (đường màu đỏ) tại hiệu điện thế $0.7 \mathrm{~V}$ (vs. $\mathrm{Ag} / \mathrm{AgCl}(\mathrm{NaCl}$ sat.), trong thời gian 10 phút.

\section{KẾT LUẬN}

Thông qua kết quả nghiên cứu, composite $\mathrm{Ni} /$ cellulose đã được tổng hợp thành công từ cellulose được tách từ vỏ cam. Các kêt quả XRD, FTIR và SEM cho thấy cellulose được tách hoàn toàn từ vỏ cam đạt kích thước 500nm và nickel bám trên ống cellulose. Các phân tích điện hóa cho thấy, composite Ni/cellulose cho kết quả tốt khi ứng dụng như là xúc tác điện hóa cho phản ứng phân hủy ethanol. Hệ xúc tác điện hóa của vật liệu composite $\mathrm{Ni} /$ cellulose duy trì cường độ dòng điện ổn định trong 10 phút ở $0.7 \mathrm{~V}(\mathrm{vs} . \mathrm{Ag} / \mathrm{AgCl}$ $(\mathrm{NaCl}$ sat.) trong môi trường $\mathrm{KOH} 0.1 \mathrm{M}$. Kết quả là một trong những đóng góp tích cực cho việc nghiên cứu và phát triển vật liệu ứng dụng trong phân hủy điện hóa ethanol.

\section{TÀI LIỆU THAM KHẢO}

[1] T. Wu, Y. Ma, Z. Qu, J. Fan, Q. Li, P. Shi, Q. Xu, Y. Min, Black Phosphorus-Graphene HeterostructureSupported Pd Nanoparticles with Superior Activity and Stability for Ethanol Electro-oxidation, ACS Appl. Mater.Interfaces. 11 (2019) 5136-5145.

[2] V. Palma, C. Ruocco, E. Meloni, F. Gallucci, A. Ricca, Enhancing Pt-Ni/CeO 2 performances for ethanol reforming by catalyst supporting on high surface silica, Catal. Today. 307 (2018) 175-188.

[3] E. Tavakolian, J. Tashkhourian, Z. Razmi, H. Kazemi, M. Hosseini-Sarvari, Ethanol electrooxidation at carbon paste electrode modified with Pd-ZnO nanoparticles, Sens. Actuators B Chem. 230 (2016) 87-93.

[4] J. Guo, R. Chen, F. Zhu, S. Sun, H.M. Villullas, Applied Catalysis B : Environmental New understandings of 
ethanol oxidation reaction mechanism on $\mathrm{Pd} / \mathrm{C}$ and $\mathrm{Pd}_{2} \mathrm{Ru} / \mathrm{C}$ catalysts in alkaline direct ethanol fuel cells, Appl. Catal. B. 224 (2018) 602-611.

[5] A.E. Fahim, R.M. Abdel Hameed, N.K. Allam, Synthesis and characterization of core-shell structured $\mathrm{M} @ \mathrm{Pd} / \mathrm{SnO}_{2}$-graphene $[\mathrm{M}=\mathrm{Co}, \mathrm{Ni}$ or $\mathrm{Cu}]$ electrocatalysts for ethanol oxidation in alkaline solution, New J. Chem. 42 (2018) 6144-6160.

[6] H. Xu, B. Yan, S. Li, J. Wang, C. Wang, J. Guo, Y. Du, N-doped graphene supported PtAu/Pt intermetallic core/dendritic shell nanocrystals for efficient electrocatalytic oxidation of formic acid, Chem. Eng. J. 334 (2018) 2638-2646.

[7] S. Fu, C. Zhu, D. Du, Y. Lin, Facile One-Step Synthesis of Three-Dimensional Pd-Ag Bimetallic Alloy Networks and Their Electrocatalytic Activity toward Ethanol Oxidation, ACS Appl. Mater. Interfaces. 7 (2015) 13842-13848.

[8] X. Yang, Q. Yang, J. Xu, C.S. Lee, Bimetallic PtPd nanoparticles on Nafion-graphene film as catalyst for ethanol electro-oxidation, J. Mater. Chem. 22 (2012) 8057-8062.

[9] T.T.K. Huynh, T.Q.N. Tran, H.H. Yoon, W.J. Kim, I.T. Kim, AgNi@ZnO nanorods grown on graphene as an anodic catalyst for direct glucose fuel cells, Korean J. Chem. Eng. 36 (2019) 1193-1200.

[10] T.Q.N. Tran, B.J. Park, W.H. Yun, T.N. Duong, H.H. Yoon, Metal-organic framework-derived Ni@C and NiO@C as anode catalysts for urea fuel cells, Sci. Rep. 10 (2020) 1-10.

[11] T. Quynh, N. Tran, S. Won, B. Ju, H. Hee, $\mathrm{CeO}_{2}$-modified $\mathrm{LaNi}_{0.6} \mathrm{Fe}_{0.4} \mathrm{O}_{3}$ perovskite and MWCNT nanocomposite for electrocatalytic oxidation and detection of urea, J. Electroanal. Chem. 818 (2018) 76-83.

[12] P.W. Menezes, A. Indra, N.R. Sahraie, A. Bergmann, P. Strasser, M. Driess, Cobalt-manganese-based spinels as multifunctional materials that unify catalytic water oxidation and oxygen reduction reactions, ChemSusChem. 8 (2015) 164-167.

[13] L. Osmieri, R. Escudero-cid, A.H.A. Monteverde, Application of a non-noble Fe-N-C catalyst for oxygen reduction reaction in an alkaline direct ethanol fuel cell, Renew. Energy.115 (2018) 226-237.

[14] Z. Guo, T. Liu, W. Li, C. Zhang, D. Zhang, Z. Pang, Carbon supported oxide-rich Pd-Cu bimetallic electrocatalysts for ethanol electrooxidation in alkaline media enhanced by $\mathrm{Cu} / \mathrm{CuOx}$, Catalysts. 6 (2016).

[15] T.Q.N. Tran, G. Das, H.H. Yoon, Nickel-metal organic framework/MWCNT composite electrode for nonenzymatic urea detection, Sens. Actuators B Chem. 243 (2017) 78-83.

[16] N.S. Nguyen, H.H. Yoon, Nickel oxide-deposited cellulose/CNT composite electrode for non-enzymatic urea detection, Sens. Actuators B Chem. 236 (2016) 304-310

[17] L. Wu, S.M.C. Ritchie, Removal of trichloroethylene from water by cellulose acetate supported bimetallic $\mathrm{Ni} / \mathrm{Fe}$ nanoparticles, Chemosphere. 63 (2006) 285-292.

[18] S.K. Mahadeva, J. Kim, Porous tin-oxide-coated regenerated cellulose as disposable and low-cost alternative transducer for urea detection, IEEE Sens. J. 13 (2013) 2223-2228.

[19] Q. Yao, B. Fan, Y. Xiong, C. Jin, Q. Sun, C. Sheng, 3D assembly based on 2D structure of Cellulose Nanofibril/Graphene Oxide Hybrid Aerogel for Adsorptive Removal of Antibiotics in Water, Sci. Rep. 7 (2017) 1-13.

[20] N.A.M. Barakat, M. Alajami, Z.K. Ghouri, S. Al-meer, Effective NiMn Nanoparticles-Functionalized Carbon Felt as an Effective Anode for Direct Urea Fuel Cells, J. Nanomater, (2018).

[21] X. Wu, W. Xing, L. Zhang, S. Zhuo, J. Zhou, G. Wang, S. Qiao, Nickel nanoparticles prepared by hydrazine hydrate reduction and their application in supercapacitor, Powder Tech. 224 (2012) 162-167.

[22] H. Yang, S. Luo, X. Li, S. Li, J. Jin, J. Ma, Controllable orientation-dependent crystal growth of high-index faceted dendritic $\mathrm{NiC}_{0.2}$ nanosheets as high-performance bifunctional electrocatalysts for overall water splitting, J. Mater. Chem. A. 4 (2016) 18499-18508.

[23] N.T.Q. Tran, H.S. Gil, G. Das, B.H. Kim, H.H. Yoon, Ni nanoparticles supported on MIL-101 as a potential catalyst for urea oxidation in direct urea fuel cells, Korean Chem. Eng. Res. 57 (2019) 387-391.

Ngày nhận bài: 05/05/2021

Ngày chấp nhận đăng: 15/07/2021 